\title{
Modelling Learners in Crowdsourcing Educational Systems
}

\author{
Solmaz Abdi $\left.{ }^{(}\right)$, Hassan Khosravi, and Shazia Sadiq \\ The University of Queensland, Brisbane, Australia \\ solmaz.abdi@uq.edu.au
}

\begin{abstract}
Traditionally, learner models estimate a student's knowledge state solely based on their performance on attempting assessment items. This can be attributed to the fact that in many traditional educational systems, students are primarily involved in just answering assessment items. In recent years, the use of crowdsourcing to support learning at scale has received significant attention. In crowdsourcing educational systems, in addition to attempting assessment items, students are engaged with other various tasks such as creating resources, creating solutions, rating the quality of resources, and giving feedback. Past studies have demonstrated that engaging students in meaningful crowdsourcing tasks, also referred to as learningsourcing, has pedagogical benefits that can enhance student learning. In this paper, we present a learner model that leverages data from students' learnersourcing contributions alongside attempting assessment items towards modelling of students' knowledge state. Results from an empirical study suggest that indeed crowdsourced contributions from students can effectively be used in modelling learners.
\end{abstract}

Keywords: Learner modelling $\cdot$ Crowdsourcing $\cdot$ Educational systems $\cdot$ Learnersourcing

\section{Introduction}

Learner models capture an abstract representation of a student's knowledge state. By and large, learner models approximate a student's knowledge state solely based on their performance on assessment items. As a point of reference, many popular learner models such as Bayesian Knowledge Tracing (BKT) [9], Item Response Theory (IRT) [24], Adaptive Factor Models (AFM) [7], Performance Factor Analysis (PFA) [25], deep knowledge tracing (DKT) [27], collaborative filtering based models [1,28], Elo-based modes [3,26], and knowledge tracing machines (KTM) [29] only use responses of students to assessment items and information about them in their modelling. This can probably be attributed to the fact that in many educational learning systems, students are prominently involved in just answering assessment items (e.g., [22]).

In recent years, the use of crowdsourcing in education, often referred to as learnersourcing [21], to support learning at scale has received significant attention. Examples of tasks that have been learnersourced include creating resources 
$[12,20]$, creating solutions and explanations [14,32], rating quality [12,20], giving feedback [23] and annotating videos [10,31]. The adoption of learnersourcing approaches is often motivated by learning theories that promote higher-order learning [4] and have been demonstrated to enhance student learning $[12,18]$.

Building on the growing evidence that learnersourcing practices enhance learning, this paper explores whether information about the learnersourcing contributions of students can be leveraged towards modelling of learners. For this exploration, we make use of the knowledge tracing machines (KTMs) framework [29] for modelling student learning. Commonly, KTMs have been used as a framework for modelling learners based on a single task (attempting assessment items). We present an encoding extension on KTMs so that the framework can capture students' interactions across multiple types of tasks (multi-tasks). To evaluate our approach, we use two data sets collected from a crowdsourcing adaptive educational system called RiPPLE in which students are engaged with multiple types of tasks within the system. Results suggest that leveraging data associated with learnersourcing contributions of the students on some types of tasks such as content creation and content moderation can be used to improve the predictive performance of the learner model compared to traditional learner models. In addition, in recent years, OLMs have been extensively integrated into various educational tools to help students in monitoring, reflecting, planning, and regulating their learning $[2,5,6,8,15,16]$. In the context of open learner models [6], updating models of the learners based on their crowdsourced contributions can further highlight the link between learnersourcing and learning as well as acknowledging their contribution. This may act as a method of incentivising student engagement with learnersourcing.

\section{Approach}

To infer learner models that incorporate data from learnersourced contributions alongside student assessment data, we present an encoding extension over the knowledge tracing machine (KTM) framework [29] so that interactions across multiple types of tasks (multi-tasks) can be captured. We denote students by $u_{n} \in\left\{u_{1} \ldots u_{N}\right\}$, learning resources (items) by $q_{m} \in\left\{q_{1} \ldots q_{M}\right\}$, and knowledge components (concepts) by $\delta_{c} \in\left\{\delta_{1} \ldots \delta_{C}\right\}$. Each item can be tagged with one or more concepts. We denote the relationship between items and concepts by $\omega_{m c} \in \Omega_{M \times C}$, where $\omega_{m c}$ is 1 if item $q_{m}$ is tagged with $\delta_{c}$, and 0 otherwise. We further denote $o_{n c}$ to keep track of the number of opportunities a student $u_{n}$ has had on a concept $\delta_{c}$ at a given time.

Commonly, KTMs have been used as a generic framework for traditional computer-based educational systems where students are only involved in attempting assessment items available in the repository of the system. Therefore, the set of tasks, $T$, represented in these systems is only limited to one task. We present a simple extension that enables KTMs to capture and encode data on students interacting with more than one task. We denote different types of tasks that students are allowed to perform in relation to items by $t_{k} \in\left\{t_{1} \ldots t_{k}\right\}$. Furthermore, 


\begin{tabular}{|c|c|c|l|c|}
\hline Students & Tasks & Resources & Concepts & Outcome \\
\hline$u_{1}$ & $t_{1}$ & $q_{3}$ & $\delta_{1}$ & 0 \\
\hline$u_{1}$ & $t_{1}$ & $q_{1}$ & $\delta_{1} \sim \delta_{2}$ & 1 \\
\hline$u_{1}$ & $t_{2}$ & $q_{2}$ & $\delta_{2}$ & 1 \\
\hline$u_{1}$ & $t_{3}$ & $q_{1}$ & $\delta_{1} \sim \delta_{2}$ & 1 \\
\hline$u_{2}$ & $t_{1}$ & $q_{1}$ & $\delta_{1} \sim \delta_{2}$ & 0 \\
\hline$u_{2}$ & $t_{1}$ & $q_{3}$ & $\delta_{1}$ & 0 \\
\hline$u_{2}$ & $t_{3}$ & $q_{3}$ & $\delta_{1}$ & 1 \\
\hline
\end{tabular}

(a)

\begin{tabular}{|c|c|c|c|c|c|c|c|c|c|c|c|c|c|c|c|}
\hline \multicolumn{2}{|c|}{ Students } & \multicolumn{2}{|c|}{ Tasks } & \multicolumn{3}{|c|}{ ||Resources } & \multicolumn{2}{|c|}{ Concepts } & \multirow{2}{*}{\multicolumn{2}{|c|}{$\frac{t_{1}}{o_{1}^{t_{1}} \mid o_{2}^{t_{1}}}$}} & \multirow{2}{*}{\multicolumn{2}{|c|}{$\frac{t_{2}}{t_{2} \mid t_{2}}$}} & \multicolumn{2}{|c|}{$t_{3}$} & \multirow[t]{2}{*}{ Outcome } \\
\hline$\overline{u_{1}}$ & $u_{2}$ & & & $\mid q_{1}$ & & $q_{3}$ & $\delta_{1}$ & $\delta_{2}$ & & & & & & & \\
\hline \begin{tabular}{|l|}
1 \\
\end{tabular} & 0 & $\begin{array}{lll}1 & 0\end{array}$ & 0 & & & $\frac{1}{1}$ & 1 & 0 & $\mid \frac{1}{1}$ & 0 & $\mid$\begin{tabular}{|l|l}
$1_{1}$ \\
\end{tabular} & $\frac{1}{2}$ & 0 & $\mid$ & 0 \\
\hline \begin{tabular}{|l|}
1 \\
\end{tabular} & 0 & \begin{tabular}{l|l|l}
1 & 0
\end{tabular} & 0 & || 1 & & 0 & 1 & 1 & $\mid$\begin{tabular}{|l|l}
$\mid 1$ \\
\end{tabular} & 0 & \begin{tabular}{|l|} 
\\
\end{tabular} & 0 & 0 & \begin{tabular}{|l|}
0 \\
\end{tabular} & 1 \\
\hline \begin{tabular}{|l|}
1 \\
\end{tabular} & 0 & \begin{tabular}{|l|l}
0 & 1
\end{tabular} & 0 & 0 & & 0 & 0 & 1 & $\mid \begin{array}{l}\mid \\
\end{array}$ & 1 & 0 & 0 & 0 & 0 & 1 \\
\hline \begin{tabular}{|l|}
1 \\
\end{tabular} & 0 & 0 & & $\| 1$ & & 0 & 1 & 1 & $\mid$\begin{tabular}{|l}
$\mid 2$ \\
\end{tabular} & 1 & $\mid$\begin{tabular}{|l|} 
\\
\end{tabular} & $\begin{array}{l} \\
\end{array}$ & 0 & 0 & 1 \\
\hline \begin{tabular}{|l|l}
0 \\
\end{tabular} & 1 & 10 & 0 & || 1 & & 0 & 1 & 1 & 0 & 0 & 0 & \begin{tabular}{|l|l|} 
\\
\end{tabular} & 0 & $|0|$ & 0 \\
\hline \begin{tabular}{|l|l}
0 \\
\end{tabular} & 1 & \begin{tabular}{l|l}
1 & 0 \\
\end{tabular} & 0 & 0 & & 1 & 1 & 0 & 1 & 0 & 0 & \begin{tabular}{|l|l|}
0 \\
\end{tabular} & 0 & 0 & 0 \\
\hline \begin{tabular}{|l|}
0 \\
\end{tabular} & 1 & \begin{tabular}{|l|l|l|} 
& 0
\end{tabular} & 1 & 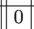 & & 1 & 1 & 0 & 2 & 0 & 0 & $\begin{array}{l}0 \\
\end{array}$ & 0 & \begin{tabular}{|l|}
0 \\
\end{tabular} & 1 \\
\hline
\end{tabular}

(b)

Fig. 1. (a) An example of a log file with 7 interactions from a crowdsourcing educational system with three types of tasks (b) On-hot encoded of the log for training KTM

we extend $o_{n c}$ to $o_{n c}^{k}$ to represent the number of opportunities a user $u_{n}$ has had on a task $t_{k}$ on a concept $\delta_{c}$ at a given time. Our proposed approach encodes and uses data from student, items, concepts, and opportunities on each of the tasks to infer a learner model $\Lambda_{N \times M}$ that estimate each student $u_{n}$ 's knowledge state for correctly attempting learning item $q_{m}$.

Figure 1 presents an example of the input file (part (a)) and its one-hot encoding (part (b)) for an educational system with multi-tasks using a chronologically ordered log file with seven observed interactions from an educational system based on two students, three tasks, three items and two concepts into a sparse vector for training KTM (for details, please see [29]).

\section{Evaluation}

Data Sets. We used two data sets obtained from an adaptive educational system called RiPPLE that recommends learning activities to students based on their knowledge state from a pool of learnersourced learning items [18]. RiPPLE enables students to create, attempt, moderate, rate, and leave comments on a range of items, including worked examples and multiple-choice questions. For this study, we consider three main types of tasks that students are allowed to perform in RiPPLE: (1) attempting items (Attempt), (2) creating new items (Create), and (3) moderating items (Moderate). The data sets are obtained from two courses, namely, 'Preparation for US Medical Licensing Examination (USMLE) (Medi) and 'Biological Fate of Drugs' (Pharm). For each of these two courses, the RiPPLE platform was used for 13 weeks of the semester. Each item in the repository is associated with one or more concepts (KC) covered in the course. Overall information about these data sets are provided in Table 1.

Table 1. overall statistics for data sets (\# stands for number of).

\begin{tabular}{l|l|l|l|l|l|l|l}
\hline Data Set & Students & Concepts & Resources & Records & \#Attempt & \#Create & \#Moderate \\
\hline Medi & 179 & 4 & 619 & 16,052 & 13,249 & 615 & 2,188 \\
Pharm & 131 & 13 & 678 & 29,982 & 28,019 & 678 & 1,285 \\
\hline
\end{tabular}


Table 2. Performance of different feature encoding for modeling learners

\begin{tabular}{l|l|l|l|l|l|l|l}
\hline ID & Model & \multicolumn{3}{l}{ Medi } & \multicolumn{3}{l}{ Pharm } \\
\cline { 3 - 8 } & & ACC & AUC & NLL & ACC & AUC & NLL \\
\hline$m_{1}$ & IRT: Student, Item & 0.698 & 0.711 & 0.567 & 0.785 & 0.771 & 0.451 \\
\hline$m_{2}$ & AFM: Student, Concept, $o^{A}$ & 0.678 & 0.599 & 0.614 & 0.772 & 0.672 & 0.504 \\
\hline$m_{3}$ & PFA: Student, Concepts, $w^{A}, f^{A}$ & 0.676 & 0.551 & 0.625 & 0.770 & 0.625 & 0.521 \\
\hline$m_{4}$ & Baseline: Student, Item, Concepts, $o^{A}$ & 0.700 & 0.713 & 0.565 & 0.784 & 0.773 & 0.453 \\
\hline$m_{5}$ & Student, Item, Concepts, $o^{A}, o^{C}, o^{M}$ & $\mathbf{0 . 7 0 7}$ & $\mathbf{0 . 7 2 3}$ & $\mathbf{0 . 5 6 3}$ & $\mathbf{0 . 7 8 8}$ & $\mathbf{0 . 7 7 8}$ & $\mathbf{0 . 4 9 9}$ \\
\hline
\end{tabular}

Models for Comparison. We implemented the proposed model based on the encoding of students, items, concepts, opportunities on attempting $\left(o^{A}\right)$, opportunities on creating $\left(o^{C}\right)$, and opportunities on moderating items $\left(o^{M}\right)$ as tasks in KTM. We compare the predictive performance of this model with traditional learner models, including IRT, AFM, and PFA. To provide a fair comparison between all models, we also made two considerations: (1) We implemented a baseline model within KTM based on the encoding of student, items, concepts, and opportunities on attempting items $\left(o^{A}\right) ;(2)$ The pairwise interaction between features in KTM is set to zero $(\mathrm{d}=0)$. For all models, we used $80 \%$ of data as the train set and predicted the outcomes on the remaining $20 \%$ as the test set. For both of the data sets, 400 epochs are used for training KTM.

Results. Table 2 compares the accuracy (ACC), area under the curve (AUC), and negative log-likelihood (NLL) of the model fit statistics related to each model. Our experimental results suggest that the learner model that leverages data related to content creation and content moderation activities $\left(m_{5}\right)$ can more accurately estimate students' knowledge state compared to its baseline and the traditional learner models that only rely on students performance on attempting learning items. This outcome is aligned with past studies on learnersourcing $[11-13,18,19,30]$ that suggest engaging students in higher-order learning impacts learning.

\section{Discussion and Conclusion}

In this paper, we presented a learner model that leveraged data from students' learnersourced contributions alongside traditional item-assessment data towards modeling the knowledge state of students in crowdsourcing educational systems. The results of our empirical studies suggest that incorporating data from students' contributions on some types of tasks associated with higher-order learning such as content creation and content moderation can be used to improve the predictive performance of the learner model. This, in turn, can improve the provided personalised feedback by the system and its adaptivity functionalities. Our findings can also have implications for learnersourcing systems that incorporate an open learner model (OLMs). Incorporating OLMs in crowdsourcing educational systems can have two benefits: 
1. Highlighting the link between learnersourcing and learning. Updating a student's competency in a concept by creating or moderating resources on that concept can help the student better associate learnersourcing with learning.

2. Acknowledging students' learningsourcing contributions. Developing models that recognise students' learningsourcing contributions and associate it with their learning may incentivise students' engagement with learnersourcing tasks, which has been identified as a challenge in learnersourcing $[17,31]$.

A major limitation of the presented evaluation is that the study was conducted on small data sets. Future directions include replicating this study across different disciplines with a larger number of students to evaluate the generalisability of our current findings.

\section{References}

1. Abdi, S., Khosravi, H., Sadiq, S.: Predicting student performance: the case of combining knowledge tracing and collaborative filtering. In: Proceedings of the International Conference on Educational Data Mining, pp. 545-548 (2018)

2. Abdi, S., Khosravi, H., Sadiq, S., Gasevic, D.: Complementing educational recommender systems with open learner models. In: Proceedings of the Tenth International Conference on Learning Analytics \& Knowledge, pp. 360-365. Association for Computing Machinery, New York (2020)

3. Abdi, S., Khosravi, H., Sadiq, S., Gasevic, D.: A multivariate Elo-based learner model for adaptive educational systems. In: Proceedings of the Educational Data Mining Conference, pp. 462-467 (2019)

4. Bloom, B.S., et al.: Taxonomy of Educational Objectives. vol. 1: Cognitive Domain, pp. 20-24. McKay, New York (1956)

5. Bull, S., Ginon, B., Boscolo, C., Johnson, M.: Introduction of learning visualisations and metacognitive support in a persuadable open learner model. In: Proceedings of the Sixth International Conference on Learning Analytics \& Knowledge, pp. 30-39. ACM (2016)

6. Bull, S., Kay, J.: Open learner models. In: Nkambou, R., Bourdeau, J., Mizoguchi, R. (eds.) Advances in Intelligent Tutoring Systems. SCI, vol. 308, pp. 301-322. Springer, Heidelberg (2010). https://doi.org/10.1007/978-3-642-14363-2_15

7. Cen, H., Koedinger, K., Junker, B.: Learning factors analysis - a general method for cognitive model evaluation and improvement. In: Ikeda, M., Ashley, K.D., Chan, T.-W. (eds.) ITS 2006. LNCS, vol. 4053, pp. 164-175. Springer, Heidelberg (2006). https://doi.org/10.1007/11774303_17

8. Cooper, K., Khosravi, H.: Graph-based visual topic dependency models: supporting assessment design and delivery at scale. In: Proceedings of the 8th International Conference on Learning Analytics and Knowledge, pp. 11-15. ACM (2018)

9. Corbett, A.T., Anderson, J.R.: Knowledge tracing: modeling the acquisition of procedural knowledge. User Model. User-Adapt. Interact. 4(4), 253-278 (1994)

10. Cross, A., Bayyapunedi, M., Ravindran, D., Cutrell, E., Thies, W.: Vidwiki: enabling the crowd to improve the legibility of online educational videos. In: Proceedings of the 17th ACM Conference on Computer Supported Cooperative Work \& Social Computing, pp. 1167-1175 (2014) 
11. Denny, P., Hamer, J., Luxton-Reilly, A., Purchase, H.: Peerwise: students sharing their multiple choice questions. In: Proceedings of the Fourth International Workshop on Computing Education Research, pp. 51-58. ACM (2008)

12. Denny, P., Luxton-Reilly, A., Hamer, J.: The peerwise system of student contributed assessment questions. In: Proceedings of the Tenth Conference on Australasian Computing Education, vol. 78, pp. 69-74. Citeseer (2008)

13. Dunlosky, J., Rawson, K.A., Marsh, E.J., Nathan, M.J., Willingham, D.T.: Improving students' learning with effective learning techniques: promising directions from cognitive and educational psychology. Psychol. Sci. Public Interest 14(1), 4-58 (2013)

14. Heffernan, N.T., et al.: The future of adaptive learning: does the crowd hold the key? Int. J. Artif. Intell. Educ. 26(2), 615-644 (2016)

15. Jivet, I., Scheffel, M., Specht, M., Drachsler, H.: License to evaluate: preparing learning analytics dashboards for educational practice. In: Proceedings of the 8th International Conference on Learning Analytics and Knowledge, pp. 31-40. ACM (2018)

16. Khosravi, H., Cooper, K.: Topic dependency models: graph-based visual analytics for communicating assessment data. J. Learn. Anal. 5(3), 136-153 (2018)

17. Khosravi, H., Gyamfi, G., Hanna, B., Lodge, J.: Fostering and supporting empirical research on evaluative judgement via a crowdsourced adaptive learning system. In: Proceedings of the 10nth International Conference on Learning Analytics and Knowledge, LAK 2020. ACM, New York (2020)

18. Khosravi, H., Kitto, K., Joseph, W.: Ripple: a crowdsourced adaptive platform for recommendation of learning activities. J. Learn. Anal. 6(3), 91-105 (2019)

19. Khosravi, H., Kitto, K., Williams, J.J.: Ripple: a crowdsourced adaptive platform for recommendation of learning activities. arXiv preprint arXiv:1910.05522 (2019)

20. Khosravi, H., Sadiq, S., Gasevic, D.: Development and adoption of an adaptive learning system: reflections and lessons learned. In: Proceedings of the 51st ACM Technical Symposium on Computer Science Education, pp. 58-64. Association for Computing Machinery, New York (2020). https://doi.org/10.1145/3328778. 3366900

21. Kim, J., et al.: Learnersourcing: improving learning with collective learner activity. Ph.D. thesis, Massachusetts Institute of Technology (2015)

22. Koedinger, K.R., Anderson, J.R., Hadley, W.H., Mark, M.A.: Intelligent tutoring goes to school in the big city (1997)

23. Kulkarni, C., et al.: Peer and self assessment in massive online classes. ACM Trans. Comput.-Hum. Interact. (TOCHI) 20(6), 1-31 (2013)

24. Lord, F.M.: Applications of Item Response Theory to Practical Testing Problems. Routledge (2012)

25. Pavlik Jr., P.I., Cen, H., Koedinger, K.R.: Performance factors analysis-a new alternative to knowledge tracing. Online Submission (2009)

26. Pelánek, R., Papoušek, J., Řihák, J., Stanislav, V., Nižnan, J.: Elo-based learner modeling for the adaptive practice of facts. User Model. User-Adapt. Interact. 27(1), 89-118 (2017)

27. Piech, C., et al.: Deep knowledge tracing. In: Advances in Neural Information Processing Systems, pp. 505-513 (2015)

28. Thai-Nghe, N., Drumond, L., Horváth, T., Krohn-Grimberghe, A., Nanopoulos, A., Schmidt-Thieme, L.: Factorization techniques for predicting student performance. In: Educational Recommender Systems and Technologies: Practices and Challenges, pp. 129-153 (2011) 
29. Vie, J.J., Kashima, H.: Knowledge tracing machines: factorization machines for knowledge tracing. In: Proceedings of the AAAI Conference on Artificial Intelligence, vol. 33, pp. 750-757 (2019)

30. Walsh, J.L., Harris, B.H., Denny, P., Smith, P.: Formative student-authored question bank: perceptions, question quality and association with summative performance. Postgrad. Med. J. 94(1108), 97-103 (2018)

31. Weir, S., Kim, J., Gajos, K.Z., Miller, R.C.: Learnersourcing subgoal labels for howto videos. In: Proceedings of the 18th ACM Conference on Computer Supported Cooperative Work \& Social Computing, pp. 405-416 (2015)

32. Williams, J.J., et al.: Axis: generating explanations at scale with learnersourcing and machine learning. In: Proceedings of the Third ACM Conference on Learning@ Scale, pp. 379-388. ACM (2016) 